\title{
Study of the Solar Magnetic Field influence on the cosmic ray Sun shadow
}

\section{Yuncheng Nan*}

Institute of Frontier and Interdisciplinary Science, Shandong University, Qingdao, Shandong 266237, China.

Key Laboratory of Particle Astrophysics, Institute of High Energy Physics, CAS, Beijing 100049, China.

E-mail: nanyc@ihep.ac.cn

\section{Cunfeng Feng}

Institute of Frontier and Interdisciplinary Science, Shandong University, Qingdao, Shandong 266237, China.

\section{Songzhan Chen}

Key Laboratory of Particle Astrophysics, Institute of High Energy Physics, CAS, Beijing 100049, China.

\section{Chaowei Jiang}

Institute of Space Science and Applied Technology, Harbin Institute of Technology, Guangdong, Shenzhen 518000, China.

\begin{abstract}
The cosmic rays originating outside the solar system are blocked by the Sun and form a shadow in the sky map. The Sun shadow is affected by the magnetic field between the Sun and the Earth. Therefore the Sun shadow can be used as a probe of the magnetic field and its intensity variations. In this work, we have developed a precise program to calculate the trajectory of each cosmic ray antiparticle to simulate the cosmic ray Sun shadow and study the influence of the magnetic field. With this program, we studied the influence of different magnetic field components between the Sun and the Earth, including the coronal magnetic field, interplanetary magnetic field, and geomagnetic field. Different magnetic field components have different influence on the shadow's displacement, extension and deficit, which is also energy dependent. In this work also checked two coronal magnetic field models, i.e., the potential field source surface (PFSS) model and the current sheet source surface (CSSS) model, their influence on Sun shadow were compared.
\end{abstract}

36th International Cosmic Ray Conference -ICRC2019-

July 24th - August 1st, 2019

Madison, WI, U.S.A.

${ }^{*}$ Speaker. 


\section{Introduction}

The Sun has a very complex magnetic field, and the variation of the magnetic field has an approximate 11- year cycle. But so far only the photosphere magnetic field is observed more accurately using the ground or satellite detector. The photosphere magnetic field is transported through the solar wind flowing continuously from the Sun, which forms the interplanetary magnetic field (IMF). The strength and direction of the IMF are only observed by the satellite at the Earth orbit. Because of the limit on the existing observation methods, the solar-terrestrial space magnetic field has still not been completely explored.

Cosmic rays originating outside the solar system usually arrive at the Earth nearly isotropically. They will be blocked by the Sun or the Moon and casts a shadow on the sky map. The positively charged nuclei are the major components of the cosmic ray. They are deflected by the Lorentz force and can be used as a probe for the magnetic field detection. Therefore, we can use the shadow to detect the whole solar-terrestrial space magnetic field and its variation[1]. The effect of the geomagnetic field(GMF) on the Moon shadow has been comprehended through measurement and simulation, and it has been taken as an important tool to check the performance of an extensive air shower array[2]. In addition, the Sun shadow has also been detected by different experiments. According to the observation[3], the Sun shadow has three basic features, including the displacement, the extension and the deficit. All these features vary depending on the phases of solar cycles, and the rigidity of cosmic rays. In order to better understand the relationships between the solar-terrestrial magnetic field and three features, and to explore the magnetic field by using the Sun shadow in the future, the simulation of the Sun shadow is essential.

In 2011, the ARGO-YBJ collaboration has quantitatively measured the IMF for the first time using the displacement of Sun shadow in the north-south direction during solar minimum in solar cycle 24[4]. In 2013, the Tibet AS $\gamma$ Collaboration checks different coronal magnetic field(CMF) models by the Sun shadow's deficit during solar minimum in Solar Cycle 23 at 10TeV [5]. And then, in 2018, they also used the displacement of Sun shadow in the north-south direction to calculate the IMF in solar cycle 23 by CSSS model and find the 1.5 times difference between it and the observed IMF[6]. In fact, the effects of different magnetic fields on the displacement and the deficit are related. They can't be discussed separately. On the other hand,in 2017, the ARGO-YBJ Collaboration reported the observation of the rigidity dependent variation of the Sun shadow in solar cycle 24 which varied from the minimum to the maximum[3]. Multiple energy points will bring more characteristics about the magnetic field.

In this work, we aim to develop a precise program to calculate the trajectory of each cosmic ray antiparticle to simulate the cosmic ray Sun shadow. And then we present the influence of different magnetic field components on the shadow's displacement, extension and deficit in solar cycle 24 in different energies. Finally, different influences of the PFSS and CSSS models on the basic features of the Sun shadow are compared.

\section{Sun Shadow Simulation}

\subsection{Magnetic field}

Large-scale CMF is modeled by extrapolating the observed photosphere magnetogram. In this 
work, magnetograms with high measurement accuracy from the Michelson Doppler Imager[7] and the Global Oscillation Network Group[8] are used in 2008 during solar maximum and 2012 during solar minimum, respectively. The PFSS[9][10] is the most popular model to calculate the CMF. It is built based on the current-free assumption between the photosphere and the source surface. The source surface is defined as an arbitrary spherical surface around the Sun. Beyond it, the magnetic field is controlled by the plasma and known as the IMF. From some comparisons with observations, the heights of the source surface are usually selected as $1.6 R_{\odot}$ [9][11] and $2.5 R_{\odot}[12]$. Another important model is the CSSS model[13]. It is built by adding a horizontal sheet current assumption. Except the source surface, the cusp surface is also involved to control the magnetic field. We choose two sets of the parameters for this model. One is the traditionally parameter of the CSSS[13]. Specifically, the height of the cusp surface(Rcp) and the source surface(Rss) equal to $2.25 R_{\odot}$ and $5 R_{\odot}$, respectively. And the length of horizontal electric currents $l_{a}$ equals to $0.2 R_{\odot}$. Another is selected by observations in solar cycle $23[14]$ with $R c p=1.7 R_{\odot}$, Rss $=10 R_{\odot}$ and $l_{a}=1 R_{\odot}$. For these two models, the order of spherical harmonics expansion $\mathrm{n}$ is set to 9 and it is enough for a description of magnetic fields.

Beyond the source surface, the basic topology of the IMF is that of an Archimedean spiral. Taking the calculated coronal magnetic field at Rss as an initial value, the IMF can be calculated by the Parker spiral model[15]. The "away" sector and the "toward" sector of the IMF can be also produced. In this model, the radial component of the solar wind velocity we get comes from OMNI observation[16]. Near the Earth, the international geomagnetic reference field-12[17] is used. The order of spherical harmonics expansion n equals 13 for the magnetic field within $600 \mathrm{~km}$ above the surface of the Earth. Over $600 \mathrm{~km}$, a dipole field is used.

\subsection{Simulation strategy}

A simulation strategy similar to the Moon shadow[2] is used in this work. We track the Sun's motion in real time and launch antiparticles isotropically in the angular range of $10^{\circ} \times 10^{\circ}$ around the Sun. This angular range is enough when the optical diameter of the $\operatorname{Sun}\left(\sim 0.5^{\circ}\right)$ and the energy of the antiparticle we used are taken into account. The antiparticle we used here represent the particle with opposite charge to cosmic ray. Then we track the position and the direction of antiparticle's motion in magnetic fields. If the antiparticle hit the Sun, a missing particle on the Sun shadow's map in the equatorial coordinate system is obtained. The principle of tracking is based on the relationship between the momentum change and the position change as $\Delta \vec{p}=\vec{F} t=q \Delta \vec{l} \times \vec{B}$. Here, we control the step size for each step of tracking by calculating the deflection angle of antiparticle in the magnetic field to avoid the errors due to step size.

Specifically, we set the launch point above the atmosphere of the Yangbajing (Tibet, China, $30.11 \mathrm{~N}, 90.53 \mathrm{E}$, altitude of $4300 \mathrm{~m}$ a.s.1.). About $10^{7}$ antiparticles are threw from the launch point. Because the deflection of the particle in the magnetic field is rigidity dependent, only the proton is simulated in this work. The energy of antiparticles distributes from $\log E=3$ to 6 in units of $\mathrm{GeV}$ with an interval of 0.5 . We track the antiparticle one by one if the zenith angle of the launch direction is between $0^{\circ}$ and $50^{\circ}$. Then we record the motion of antiparticles hitting the Sun and the Sun shadow's map. 


\section{Influences of Different Magnetic Fields on the Sun Shadow}

The large-scale CMF, the IMF and the GMF occupy different positions in the solar-terrestrial space and they have different directions and strengths. In order to calculate the influences of every magnetic field on the Sun shadow accurately, each magnetic field is added respectively to the simulation program mentioned above. Because the GMF has a limited range and the Sun and the Moon have almost the same optical diameter, we found the GMF moves the Sun shadow at $1 \mathrm{TeV}$ westward by about $1.46^{\circ}$ which is almost the same as the result from the Moon shadow[2].

\subsection{Coronal Magnetic Field}

The influences from the CMF on the Sun shadow are complicated. To explore the relationship between the CMF and the Sun shadow, Simulated Sun shadows which are influenced only by the PFSS model in 2008 are shown in Figure 1. In these three panels, the source surface radii is $1.6 R_{\odot}$. At $1 \mathrm{TeV}, 10 \mathrm{TeV}$, and $100 \mathrm{TeV}$, Sun shadows appear in the obvious Sun's position. Also, the extension is observed in two low-energy maps slightly.
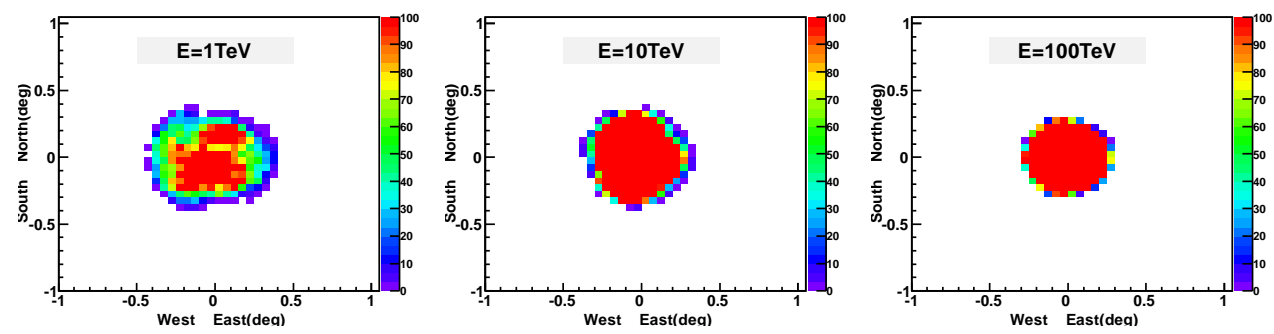

Figure 1: Energy variations of counts maps of the simulated Sun shadow between $1 \mathrm{TeV}$ and $100 \mathrm{TeV}$ in 2008.

We use the deficit ratio to quantitatively describe the deficit of the Sun shadow. The deficit ratio is defined as the result of the number of antiparticle hitting the Sun with magnetic fields divided by that without magnetic fields. The deficit ratios at different energies are shown in Figure 2. With the blue dots in Figure 2, we found that the value of the deficit ratio increases firstly, reaches its maximum at $10 \mathrm{TeV}$ and then decreases to $100 \%$ with the increase of energy. In addition, other magnetic field's results is added to comparison in Figure2. The red, green and black dots represent

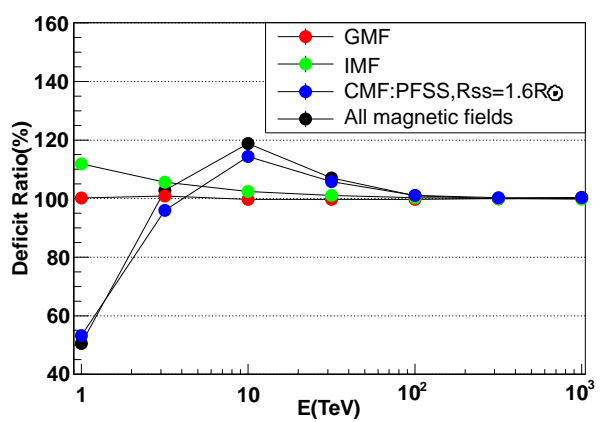

Figure 2: Energy variations of the deficit ratio for different magnetic fields in 2008. The red, green, blue and black dots represent the calculated deficit ratios of the simulated Sun shadow assuming the GMF, the IMF, the CMF, and the whole magnetic fields. The PFSS model with $\mathrm{Rss}=1.6_{\odot}$ is used for the $\mathrm{CMF}$. 
the deficit ratios from the GMF, the IMF and the whole magnetic field, respectively. The deficit ratios influenced by the GMF and the IMF are much lower than that from the PFSS. Furthermore, by comparing the trend of the deficit ratios from the whole magnetic field and from the PFSS with energy, we can conclude that the CMF mainly affects the deficit ratio of the Sun shadow.
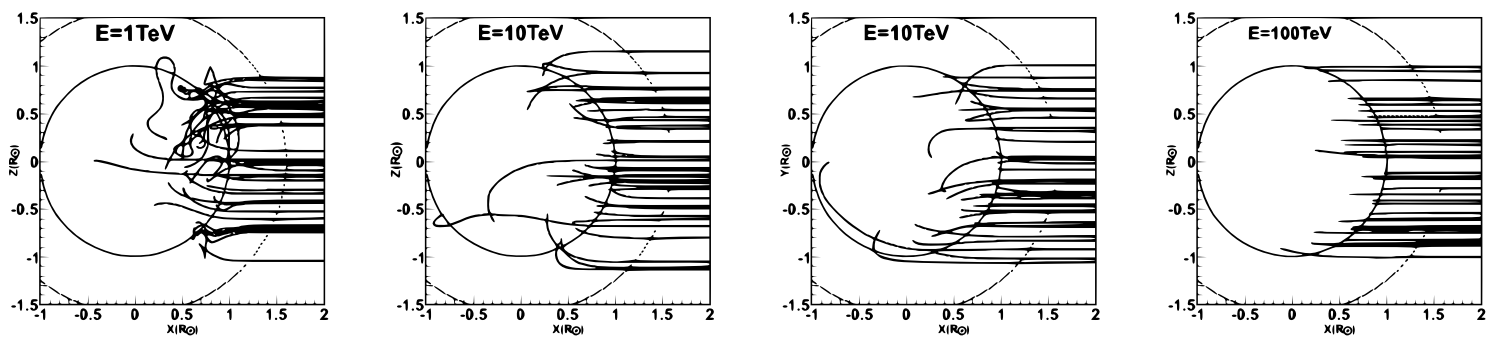

Figure 3: Moving trails of antiparticles hitting the Sun in PFSS model in heliocentric earth ecliptic (HEE) coordinates in 2008. The four panels represent the simulated trials which is projected on the different coordinate plans at $1 \mathrm{TeV}, 10 \mathrm{TeV}$ and $100 \mathrm{TeV}$. The small and the large circles in each panel represent the Sun and the source surface, respectively.

To explain how the CMF affects the deficit ratio of the Sun shadow, we show the moving trails of antiparticles hitting the Sun in the heliocentric earth ecliptic (HEE) coordinate system in Figure 3. At $1 \mathrm{TeV}$, the $\mathrm{CMF}$ can deflect the antiparticles at large angles and decrease the value of the deficit ratio. At $10 \mathrm{TeV}$, the $\mathrm{CMF}$ can be seen easily from the trials because of the high energy and the small deflection angle. The magnetic fields which are in the direction of the HEE-y axis for the two poles and in the direction of the HEE-z axis for the equator deflect the antiparticle which are not emitted toward the Sun initially and make them hit the Sun finally. Therefore, the deficit ratio exceeds $100 \%$. At $100 \mathrm{TeV}$, the antiparticles can hardly be deflected by the CMF and all of them hit the Sun. And the corresponding deficit ratio calculated equals to $100 \%$.

\subsection{Interplanetary Magnetic Field}

From the calculation in the last section, we know that the influence from the IMF on the Sun shadow's deficit ratio is weak. At $1 \mathrm{TeV}$, the corresponding maps of the Sun shadow which is influenced only by the IMF is shown in the panel (a) in Firgure4. There is no obvious displacement of the centre of the Sun shadow. But the Sun shadow is extended by the IMF and has an asymmetric $\mathrm{X}$ shape.

To comprehend the formation of the Sun shadow, the one-year Sun shadow is separated according to the different Carrington rotations. We found, for the first time, the Sun shadow is seasonal
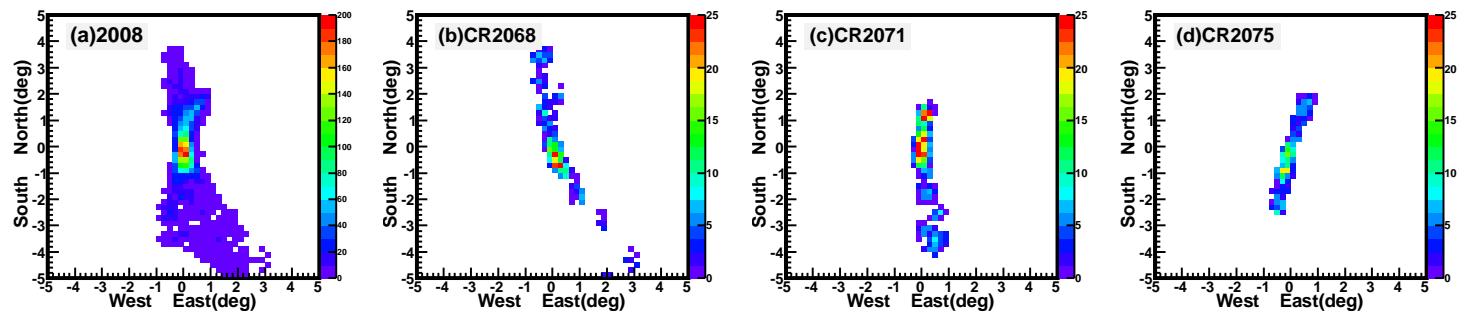

Figure 4: Counts maps of the Sun shadow influenced only by the IMF. The panel (a), (b), (c) and(d) refer to the Sun shadow in 2008 , CR2068, CR2071 and CR2075, respectively. 
dependence. The panel (b), (c) and (d) in Figure4 present the Sun shadow in CR2068, CR2071, CR2075. And these three Carrington rotations correspond to the day near the Spring Equinox, the Summer Solstice and the Autumnal Equinox in the northern hemisphere, respectively. The Sun shadow in winter is out of our field of view. In the panel (b), (c) and (d), the Sun shadow tilt to the left, the middle and the right in different seasons. The reasons of these results are the latitude of the Yangbajing and the obliquity of the ecliptic. Near Summer Solstice, the angles between the directions of antiparticle emission and the IMF's equatorial plane are small. The "away/toward" sectors of the IMF mainly move the shadows to the northward/southward and make the Sun shadow extend in the north-south direction. Near Spring Equinox, the angles are large and the "away/toward" sectors deflect the shadows to the westward/eastward additionally. Near Autumn Equinox, the tilt of the Sun shadow is opposite in the equatorial coordinate system. When Sun shadows of each Carrington rotation are added up, an asymmetric X-shaped Sun shadow is formed.

In conclusion, the GMF, the CMF and the IMF are the main reasons for the Sun shadow's displacement to the westward, deficit, and extension in the north-south direction, respectively.

\section{Influences of Different CMF's Models on the Sun Shadow}

In this section, a more realistic Sun shadow which is deflected by the magnetic field in the whole solar-terrestrial space is simulated. We use basic characteristics of the Sun shadow to compare different parameters and different coronal large-scale magnetic field's models.

The parameters of the PFSS and the CSSS model have been tested by some observed methods before solar cycle 24 . In solar cycle 24 , some simple comparisons between the magnetic fields near the Earth which come from the OMNI observation and that from models predictions are given firstly. In Figure5, the Bx components of the field are compared for CR2070 during solar minimum and CR2123 during solar maximum, respectively. Also, $\chi^{2}$ test is used to quantitative comparisons in Table1. In CR2070, the height of the source surface should be lower than $2.5 R_{\odot}$ for the PFSS model because of higher possibility. Due to the current sheet in the CSSS model, the values of Bx vary sharply as time goes by. For the CSSS model, the $10 R_{\odot}$ is a better choice for Rss. In CR2123,
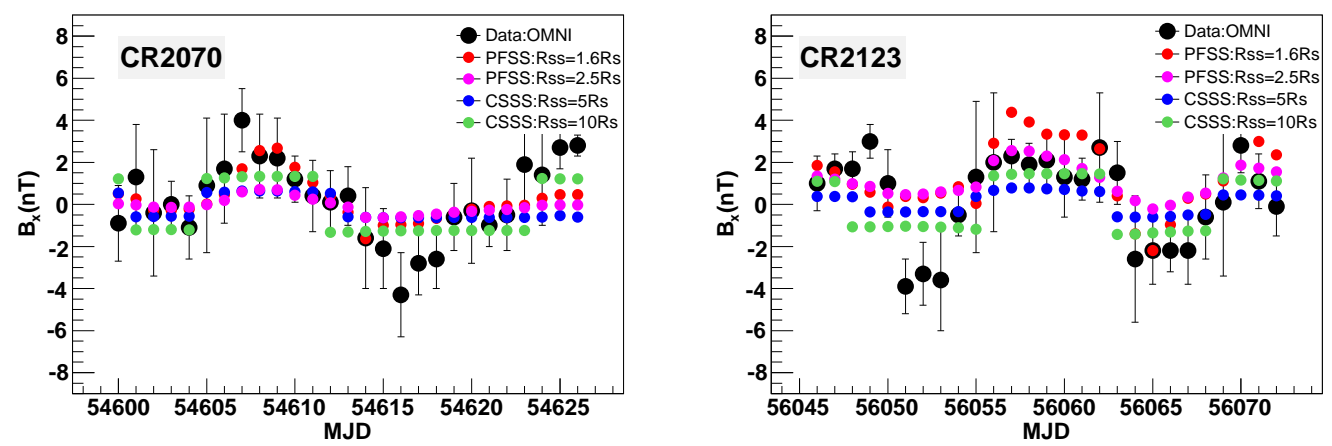

Figure 5: Comparisons between the measurement and the model calculation of the IMF at 1AU using different models and parameters in CR2070 and CR2123. The solid dots represent the measurements of the field component $B_{x}$ using the OMNI observational data. The error bars along the y-axis indicate the RMS of the $B_{x}$. The red, pink, blue and green dots represent the calculated Bx using PFSS (Rss=1.6 $R_{\odot}, 2.5 R_{\odot}$ ) and $\operatorname{CSSS}\left(\mathrm{Rss}=5 R_{\odot}, 10 R_{\odot}\right)$, respectively. 
Table 1: $\chi^{2}$ test between the measurement and the model calculation of the IMF's $B_{x}$ and $B_{y}$ components near the Earth.

\begin{tabular}{ccccc}
\hline & \multicolumn{2}{c}{ CR2070 } & \multicolumn{2}{c}{ CR2123 } \\
& $\chi^{2} / \mathrm{DOF}$ & $\mathrm{p}$-value & $\chi^{2} / \mathrm{DOF}$ & $\mathrm{p}$-value \\
\hline PFSS Rss $=1.6 R_{\odot}$ & $68.595 / 54$ & 0.087 & $122.017 / 54$ & $4.970 \times 10^{-9}$ \\
\hline PFSS Rss $=2.5 R_{\odot}$ & $90.990 / 54$ & 0.001 & $105.811 / 54$ & $8.310 \times 10^{-7}$ \\
\hline CSSS Rss $=5 R_{\odot}$ & $109.074 / 54$ & $1.357 \times 10^{-5}$ & $140.604 / 54$ & $9.032 \times 10^{-12}$ \\
\hline CSSS Rss $=10 R_{\odot}$ & $62.877 / 54$ & 0.191 & $145.211 / 54$ & $1.781 \times 10^{-12}$ \\
\hline
\end{tabular}

there is a big difference between model and observation. Just the PFSS model with Rss equals to $2.5 R_{\odot}$ have a slightly higher possibility.

All of the above comparisons are limited. Then the extensions and the deficit ratios of Sun shadows for different magnetic models are calculated at different energies to compare models. The value of extension is obtained by the RMS of the Sun Shadow's map which is projected in the north-south direction. The results are shown in Figure6.
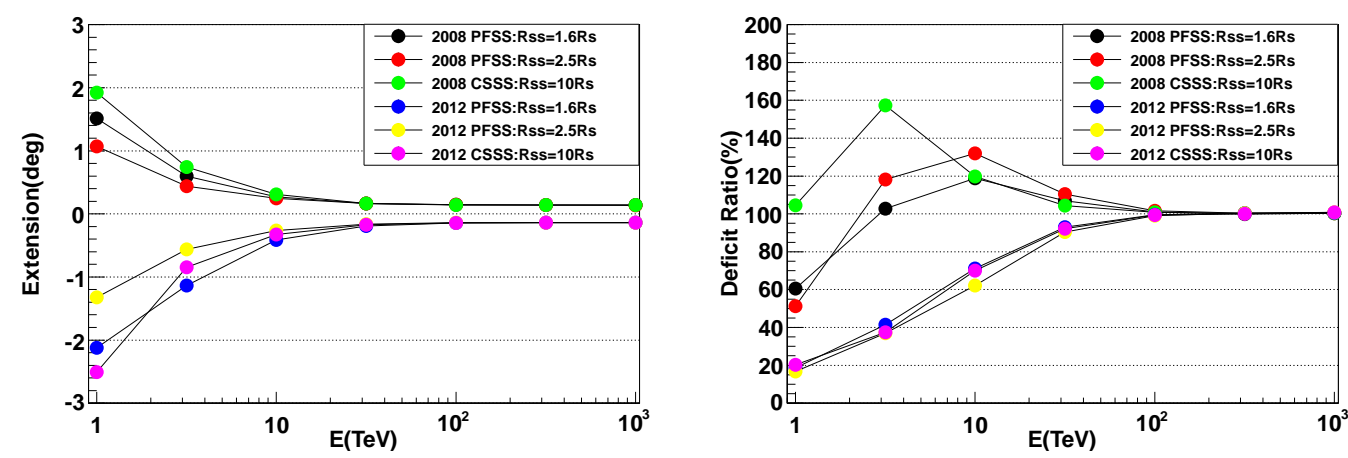

Figure 6: Comparisons of the Sun shadow's extension and deficit ratio between different CMF's models and parameters at different energies, respectively. The black, red and green dots represent results from the PFSS(Rss $\left.=1.6 R_{\odot}, 2.5 R_{\odot}\right)$ and $\operatorname{CSSS}\left(10 R_{\odot}\right)$ in 2008, respectively. And the blue, yellow, pink dots represent results in 2012. For the extension in 2012, additional negative values are used for easy viewing.

In 2008, below $10 \mathrm{TeV}$, differences caused by models and parameters are obvious by comparing the extension and the deficit ratio. For the PFSS model, the lower height of the source surface will increase the extension of the Sun shadow. And the current sheet also enables the CSSS model to possess this ability. At $10 \mathrm{TeV}$, the deficit ratio calculated by the PFSS(Rss $\left.=2.5 R_{\odot}\right)$ is obviously larger than that calculated by the $\operatorname{CSSS}\left(10 R_{\odot}\right)$. This result is contrary to that from Tibet AS $\gamma$. The difference between our results may comes from the different magnetograms, different time ranges or something else. When the height of the source surface decrease, the deficit ratios of the $\operatorname{CSSS}\left(10 R_{\odot}\right)$ and the $\operatorname{PFSS}\left(1.6 R_{\odot}\right)$ are almost the same. We can see that parameters have a great impact on the deficit ratio of the Sun shadow. That means more simulation in different and reasonable parameters is required if we want to use the Sun shadow to study the CMF in the future.

In 2012, differences between the results from models and parameters only can be seen at the extension of the Sun shadow below 10TeV. As the results in 2008, the height of the source surface in PFSS can determine the extension of the Sun shadow. But the CSSS model doesn't increase the extension at about $3.16 \mathrm{TeV}$ as it did in 2008. To explore more about the structure of the coronal 
large-scale magnetic field results, the experimental data and more simulation are necessary in the future.

\section{Summary}

Through the simulation, we found that the CMF and the IMF mainly affect the deficit ratio and the extension of the Sun shadow, respectively. For the first time, we see the seasonal dependence of the extension of the Sun shadow. To explore more about the CMF, different models and parameters are compared by using the deficit ratio and the extension of the Sun shadow. We found that different parameters in the PFSS model will give a different result which is used to check models. In the future, more simulation and the experimental data from the ARGO-YBJ and the LHAASO will be added to check models and choose parameters.

\section{Acknowledgement}

This work is supported by the National Key R\&D Program of China (No. 2018YFA0404201) and the Natural Sciences Foundation of China (No. 11575203 and No. 11775153).

\section{References}

[1] G. W. Clark, 1957, Phys. Rev. 108, 450

[2] B. Bartoli, P.Bernardini et al, 2011, PRD, 84, 022003

[3] S. Z. Chen, Y. C. Nan, 2017, 35nd ICRC

[4] G. Aielli, C. Bacci et al, 2011, ApJ, 729, 113

[5] M. Amenomori, X. J. Bi et al, 2013, PRL, 111, 011101

[6] M. Amenomori, X. J. Bi et al, 2018, PRL, 120, 031101

[7] MDI: http://soi.stanford.edu/

[8] GONG: https://gong.nso.edu/

[9] K. H. Schatten, J. M. Wilcox et al, 1969, SoPh, 6,442

[10] M. D. Altschuler, G. J. Newkirk, 1969, SoPh, 9,131

[11] C. O. Lee, J. G. Luhmann et al, 2011, SoPh, 269,367

[12] J. T. Hoeksema, 1984, PhD thesis, Stanford Univ

[13] X. P. Zhao, J. T. Hoesksema, 1995, Jour. Geophys. Res, 100,19

[14] M. Schüssler, I. Baumann, 2006, A\&A 459, 945

[15] E. N. Parker, 1958, ApJ, 128, 66

[16] OMNI: https://omniweb.gsfc.nasa.gov

[17] IGRF: https://www.ngdc.noaa.gov/IAGA/vmod/igrf.html 